\title{
Le social local: un nouvel enjeu pour les villes
}

\author{
Pierre-André Tremblay, Ph.D. \\ Université du Québec à Chicoutimi
}

\section{Introduction : un cas concret}

La réunion, comme les autres, dure un peu plus de trois heures. Réunir cette vingtaine de personnes à chaque cinq ou six semaines depuis quatre ou cinq ans était déjà un succès. Ce sont tous des gens occupés, qui travaillent sur de nombreux dossiers et souvent avec des moyens très réduits. Le «milieu communautaire» est très majoritaire : centres communautaires, associations de locataires, organismes œuvrant avec les jeunes, services budgétaires, groupes travaillant à la sécurité alimentaire, Corporation de développement communautaire - la plupart des secteurs sont représentés. Mais on retrouve aussi autour de la table des fonctionnaires provinciaux (Sécurité du revenu ou de la Solidarité sociale), municipaux (service des loisirs et, parfois, de l'urbanisme) et, étrangement, un universitaire. D'autres sont venus à des réunions précédentes : groupes de défense des personnes assistées sociales, groupes environnementaux, représentants du Centre local d'emploi (CLE) ou du Centre local de développement (CLD); ils reviennent encore parfois, lorsque leur horaire le permet. D'autres ne reviennent plus, et leur absence est notable; c'est le cas des représentants des milieux d'affaires, dont la présence n'a jamais fait l'unanimité et a parfois soulevé des débats assez âpres.

Les principes de participation à la Table de concertaction sur la lutte à la pauvreté n'ont jamais été vraiment clairs. Il y a une part d'auto-sélection : il faut être accepté par les autres et se sentir à l'aise avec eux, se sentir concerné par la question de la pauvreté et y agir. Un trait commun à tous les participants est qu'ils représentent des "organismesterrain ", selon leurs termes, des organisations ou des personnes ouvrant directement avec les personnes pauvres. L'autre trait frappant est l'absence des pauvres eux-mêmes : la Table ne regroupe que des intervenantes ${ }^{1}$.

Si elle rassemble essentiellement des personnes appartenant à des groupes de la société civile, la Table origine d'une recherche supportée par un organisme du gouvernement fédéral qui désirait promouvoir le développement économique communautaire au sein des quartiers en dévitalisation de Chicoutimi ${ }^{2}$. Il ne s'agit donc pas d'un regroupement spontané et autonome. On peut en déduire que les actions de la société civile s'articulent à celles de la société politique plus qu'elles ne s'y opposent. La question est plutôt de savoir comment s'y articuler : l'enquête faisait nettement ressortir le manque d'enthousiasme des intervenantes rencontrées à l'égard de la mise sur pied d'une nouvelle organisation. L'idée d'une Table de concert-action émergea ainsi : une structure souple, presque informelle, visant essentiellement à échanger des informations, à mettre en commun des analyses et des questionnements, contribuant à l'auto-formation des participantes et servant à dépasser les limites de leurs actions isolées. Mais afin d'éviter la superficialité qui marque beaucoup des tables de concertation, il fut décidé que la coordination des informations se ferait dans l'action plutôt que seulement dans la discussion. Il fallait agir, pas seulement discourir.

Cependant, les premiers temps consistèrent surtout en une familiarisation des participantes, des présentations des groupes et de leurs actions, etc., sous la supervision de l'équipe universitaire. L'objectif était de tisser des liens allant au-delà de la représentation formelle et de créer un langage commun aux participantes afin que puisse se créer un espace délibératif partagé. Cette étape fut franchie quand le CLSC 
accepta de déléguer deux organisateurs communautaires pour animer la Table, conjointement avec la Corporation de développement communautaire du Roc $^{3}$. En ces temps de ressources rares, cela démontre l'intérêt que souleva la Table.

Le fonctionnement de la Table se formalisa au même moment. Des sous-comités furent organisés, dont la tâche était d'animer des actions sur des thèmes particuliers : transport en commun, sécurité alimentaire, loisirs et sécurité, etc. Au vu de la mission que s'était donnée la table - lutter contre la pauvreté - il est clair que ces sujets touchent moins les causes de la pauvreté que les conditions de vie des personnes pauvres. D'autre comités se rapprochent plus de la compréhension classique de la lutte à la pauvreté : un comité mit sur pied un programme d'insertion au travail de personnes prestataires de la sécurité du revenu, contribua à leur sélection, les aida et les supporta, avec un taux de succès appréciable. Une autre intervention de la Table, qui demanda beaucoup d'énergie, fut l'organisation d'un forum dans le secteur ouest de la ville, une zone particulièrement marquée par les signes habituels de la pauvreté et où des intervenantes avaient mis sur pied, quelques années auparavant, le Centre communautaire St-Paul. Le forum, qui s'adressait à la population plus encore qu'aux intervenants sociaux, visait à faire le point sur la situation du quartier : emploi, logement, transport, services collectifs, loisirs, personnes âgées, jeunes et adolescents, etc. Il est une belle illustration de l'approche de quartier qui a été peu à peu privilégiée par la Table et dont le trait fondamental est d'aborder les problèmes de pauvreté, non pas en ciblant des catégories de personnes mais en identifiant des zones sur lesquelles les intervenantes joindront leurs efforts.

\footnotetext{
Nos actions les plus minuscules se déroulent dans un univers marqué par les changements macrostructurels : la mondialisation qui domine notre époque a des répercussions dans les pores les plus infimes des sociétés.
}

La rapidité et l'enthousiasme avec lesquels beaucoup de groupes acceptèrent de participer indiquent que le temps était sans doute venu d'un tel lieu de discussion et de mise en commun. Leur persévérance (huit rencontres par année depuis cinq ans) montre que le be- soin s'en fait toujours sentir. Pourquoi ? Les sections suivantes s'appuieront sur l'idée qu'un trait fondamental des changements que connaît le Québec, comme d'autres sociétés industrialisées, est un déplacement du lieu de gestion du social. Le fordisme ${ }^{4}$, et l'État-providence qu'il s'était donné, avait fait de l'État-nation l'échelle première de définition et, donc, de gestion des problèmes sociaux. L'épuisement de ce modèle semble amener à l'avant-scène le palier local, et cela paraît se faire sans le réduire à l'appareil municipal. Au contraire : la société civile locale en est un acteur essentiel.

La première section de cet article présente quelques aspects fondamentaux des transformations en cours. La section suivante explore la question de la pauvreté, alors que la troisième s'arrête au mode de fonctionnement de cette société civile locale, en s'appuyant sur l'exemple de la Table de lutte à la pauvreté de Chicoutimi. Avant la conclusion, qui insiste sur l'importance de la démocratie locale, la dernière section considérera quelques enjeux inhérents au développement économique communautaire et au développement social.

\section{Les transformations sociales}

Nos actions les plus minuscules se déroulent dans un univers marqué par les changements macrostructurels : la mondialisation qui domine notre époque a des répercussions dans les pores les plus infimes des sociétés. L'accélération des échanges financiers en est sans doute le trait le plus évident, mais elle s'accompagne de déplacements toujours plus rapides et nombreux des idées et des personnes. Il ne s'agit plus uniquement des simples processus de diffusion d'un centre vers les périphéries, mais d'une structuration réticulaire en couches multiples ${ }^{5}$. Il ne s'agit pas d'une abolition des frontières, mais d'un changement de leurs fonctions; elles sont des lieux de négociation des responsabilités et plus seulement des clôtures définissant un espace clos. Dans ce contexte, la mobilité devient une dimension constitutive du lien social ${ }^{6}$. L'époque est à la migration, au métissage, à la flexibilité, qui impliquent aussi de l'insécurité et du risque, comme le rappelle Beck ${ }^{7}$.

Il s'agit d'un renversement important de la tendance prise par les sociétés développées depuis une centaine d'années. Elles avaient recherché la stabilité et la 
sécurité ${ }^{8}$, produisant des cultures axées sur l'identité nationale, des institutions d'un formidable degré de bureaucratisme, des modes d'organisations privilégiant les accords au sommet et à long terme, des processus productifs planifiés et rigides. Sous la pression des mouvements sociaux, cela avait exigé et produit une réduction de l'espace d'incertitude associé au marché ${ }^{9}$, auquel on avait accolé des modes d'allocation organisés selon les liens politiques: à l'économie privée typique du capitalisme «classique » s'était ajoutée une économie publique chargée d'en amenuiser les principales aspérités. D'une certaine façon, cela revenait à reconnaître à la vie sociale une légitimité et une rationalité face à la vie économique.

Devenu l'institution fondamentale du fordisme, l'État social s'est trouvé mis à mal par la porosité des frontières exigée par le redéploiement du capitalisme à partir des années 1970, qui favorise une indépendance des entreprises à l'égard des conditions locales et nationales, ainsi que par l'écart entre ses responsabilités et ses moyens. Il dut chercher un réajustement, qu'il ne semble pas avoir encore tout à fait trouvé et que désignent les termes connus : néo-libéralisme ${ }^{10}$, décentralisation ${ }^{11}$, réingénierie ${ }^{12}$. La dominante est à voir dans le marché le mode d'allocation le plus rationnel, auquel devront s'ajuster les autres. Cela affecte particulièrement les programmes sociaux qui gênent la flexibilité exigée par la concurrence mondiale, qui sont trop coûteux (ce qui remet en question la profitabilité des entreprises) et qui offrent trop d'autonomie à l'égard des exigences du marché du travail. Le résultat est une société à économie solide, mais où les dimensions sociales sont réduites à la portion congrue. Certes, le PIB croît de façon relativement élevée, la création d'emplois est bonne, l'apparition et la survie des entreprises se produisent à un rythme soutenu, etc. Cependant, les inégalités sociales croissent, la concentration de la richesse est de plus en plus notable, une frange importante de la population se retrouve exclue des retombées de la croissance, les emplois créés sont de plus en plus « atypiques » (à durée déterminée, précaires, à temps partiel, etc. $)^{13}$.

Tous ces traits amènent une fragilisation de la cohésion, un effilochement du tissu social qui, outre les problèmes d'anomie qu'il provoque, risque de remettre en question la croissance économique elle-même. Pendant le fordisme, c'est à l'État que revenait la

fonction de maintien de la cohérence des interactions, mais l'État post-fordiste n'ayant pas la même capacité, la question de la gouvernance se pose avec de plus en plus d'acuité. Le terme désigne les interrelations entre l'État et ses divers appareils, comme instruments essentiels de l'action de la société sur elle-même, et la société civile.

C'est dans ce contexte que se pose la question du social, qui ne désigne donc pas tout ce qui fait partie de la vie en société mais essentiellement les aspects extra-économiques et extra-politiques de la vie des personnes, des groupes et des collectivités. On peut retenir trois dimensions plus vivement ressenties : la définition des problèmes sociaux et la pauvreté, puis la question de la mise en œuvre des efforts de développement social, ce qu'on abordera en considérant le mode de fonctionnement développé par les efforts de la Table de lutte à la pauvreté. Troisièmement, il faut considérer les enjeux soulevés par l'approche en termes de développement économique communautaire.

\section{Les problèmes sociaux ne s'imposent pas d'eux-mêmes; ils apparaissent, disparaissent, se transforment, bref, ils sont une construction sociale, ce qui implique que le premier enjeu est de faire reconnaître leur existence et le second, leur mode de structuration.}

\section{Les problèmes sociaux et la pauvreté}

En pratique, compte tenu de l'hégémonie de l'approche économiciste, la question du social se pose d'abord sous la forme de problèmes sociaux, dysfonctions gênant le fonctionnement harmonieux des échanges marchands: criminalité, violence, éclatement familial, comportements anomiques, santé, perte du sentiment d'appartenance, pauvreté et exclusion, ces deux derniers aspects soulevant particulièrement l'inquiétude ${ }^{14}$. Évidemment, les problèmes sociaux ne s'imposent pas d'eux-mêmes; ils apparaissent, disparaissent, se transforment, bref, ils sont une construction sociale ${ }^{15}$, ce qui implique que le premier enjeu est de faire reconnaître leur existence et le second, leur mode de structuration.

C'est là une des principales difficultés rencontrées par la Table de lutte à la pauvreté de Chicoutimi. Les 
premières consultations ${ }^{16}$ avaient mis à jour les nombreuses significations que le terme peut avoir : simple insuffisance de revenus ? Dénuement complet ? Temporaire ? Permanent? Se limite-t-elle à des aspects financiers ou recouvre-t-elle des dimensions touchant aux relations sociales des personnes, c'est-à-dire à l'exclusion ? Est-elle une propriété des personnes, des familles ou des groupes? Quels groupes : culturels ? Ethniques? Spatiaux? Générationnels ? La mise au clair de ces interprétations, sans même parler de leur conciliation, est une question délicate et lourde de conséquences.

La difficulté augmente lorsqu'on tente de circonscrire les causes et les conséquences de la pauvreté (quelle qu'en soit la définition), c'est-à-dire qu'on dépasse le strict niveau définitionnel pour considérer l'écheveau des interactions qu'elle enclenche. Il apparaît rapidement que la pauvreté est liée à des dimensions éducationnelles et à des compétences techniques (ou à leur absence), mais il faut ajouter qu'elles n'ont de sens que dans un certain état du marché du travail; on peut avoir des compétences, mais ne pas trouver à les utiliser pour obtenir un revenu suffisant. Il ne suffit donc pas d'augmenter l' «employabilité » des personnes. De plus, il faut que les emplois rendus accessibles par ces compétences soient disponibles et qu'ils permettent de sortir de la pauvreté. Bref, il ne faut pas considérer la personne isolément, mais dans son rapport au marché du travail.

Par ailleurs, la pauvreté est liée à des traits personnels qui peuvent être acquis (comportements, habitudes, choix éthiques, localisation, situation de santé mentale ou physique) ou non (âge, genre, race, etc.). Cette énumération fait rapidement apparaître que la question est au-delà de la personne et tient au groupe à laquelle elle est assimilée. On ne peut donc pas se borner à blâmer les individus en situation de pauvreté, mais œuvrer sur les stigmates et stéréotypes qu'on leur impose. Et se demander si les notions de cause et de conséquence ont vraiment du sens : est-on pauvre parce que malade, ou malade parce que pauvre?

Ces considérations n'ont rien de théorique mais influent immédiatement sur les modalités d'intervention : faut-il avoir des « groupes-cibles »? Lesquels? Doit-on viser les personnes ? Les groupes ? Les catégories sociales? Comment les aborder? Comment élaborer des stratégies qui maximisent l'impact, en ces temps de ressources rares? La Table de Chicou- timi, après d'assez longues hésitations, opta pour une approche de quartier et choisit de viser non des catégories ou des personnes, mais des espaces particulièrement défavorisés où œuvraient déjà certains des participants. Le secteur ouest fut particulièrement ciblé. Il correspond à certains des espaces défavorisés de la zone urbaine centrale de Chicoutimi que permet d'identifier la figure 1.

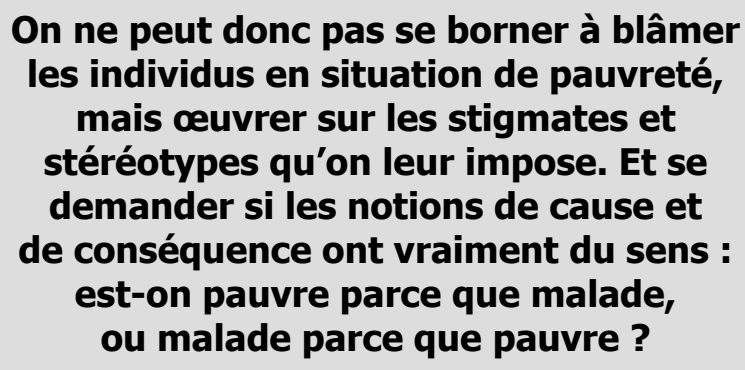

\section{Le mode de fonctionnement}

Le choix d'une approche spatialement définie est intéressant à plus d'un titre. Elle permet d'éviter de stigmatiser des personnes, ce qui est indispensable car la honte d'être identifié comme pauvre est au cœur des difficultés d'intervention. Elle favorise une transversalité, ce qui permet d'envisager des complémentarités entre les expertises présentes, ce qui est cohérent avec l'aspect multidimensionnel de la pauvreté. De façon symétrique, cela peut permettre de contourner les cercles vicieux entre les causes et les conséquences auxquels on a fait allusion plus haut. De plus, et cela ne doit pas être sous-estimé, cette approche respecte les «chasses gardées » de chacune, car la compétence reconnue est aussi, pour les divers intervenants, un gage d'autonomie, voire une garantie de survie. Sous la nécessité évidente d'éviter les dédoublements se cache donc fréquemment une stratégie de survie organisationnelle, ce qui ne surprend guère quand on sait combien les intervenants du social (groupes communautaires, organismes parapublics et même employés du secteur public) doivent lutter pour conserver le peu de ressources qui leur sont concédées.

Une autre explication de ce choix est moins évidente. Elle repose sur le point de vue implicite que la lutte à la pauvreté ne peut se faire qu'en privilégiant les aspects collectifs de la vie des personnes. Cela signifie, bien sûr, que la pauvreté est un phénomène social 
tant dans ses causes que dans ses manifestations, mais aussi que seule la mise en commun des efforts pourra $\mathrm{y}$ faire quelque chose. Par ailleurs, on peut y retrouver un choix normatif: la vie en commun, l'adhésion au groupe sont bonnes, le chacun-pour-soi mauvais. C'est là un des sens du terme communauté : le collectif, en ce qu'il a de positif ${ }^{18}$. La vie normale, de ce point de vue, est une vie incluse dans un groupe. $\mathrm{Ce}$ qui favorise, provoque ou induit l'individualisme, mène à terme à l'exclusion et doit être modifié. Une des activités principales de la Table contre la pauvreté fut l'organisation d'un «Forum du secteur ouest», dont le but avoué était de favoriser la genèse d'un sentiment d'appartenance à un quartier pratiquement dénué d'identité. Par des rencontres et des discussions, par des débats et des échanges, mais aussi par une fête où les enfants ont une place de choix, on veut faire apparaître une communauté là où n'existent que des familles sans liens les unes avec les autres. Ici, l'esprit communautaire n'est pas tant le préalable de l'action sociale que sa conséquence.

Figure 1 - Indice de défavorisation ${ }^{17}$

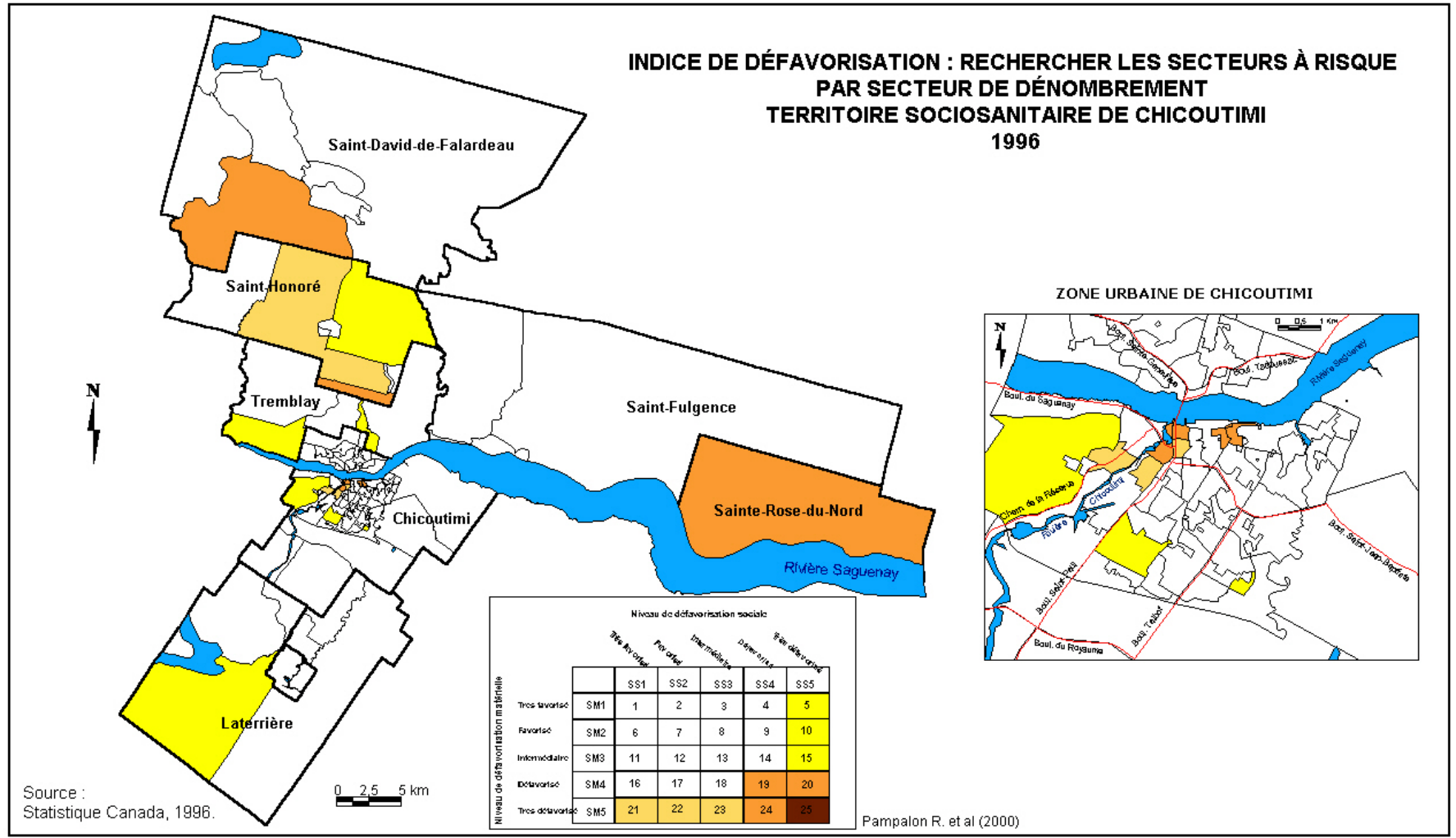

L'approche de quartier n'exige donc pas que l'espace visé ait une existence sociale précédant l'intervention. Par contre, si elle veut avoir quelque succès, celle-ci doit nécessairement tenir compte des caractéristiques objectives de la situation. Où se termine, où commence le quartier? Quels seront les critères délimitant ses frontières? La délimitation de ce qu'il faut entendre par « secteur ouest » a ainsi dû tenir compte des marqueurs spatiaux (rues, boulevards) et architecturaux (types de logements), mais les plus fondamentaux furent les aspects sociaux : composition des fa- milles, niveaux de revenus des habitants et leur source, durée de leur séjour, etc. et, ce qui est nécessairement intangible, l' " image » du secteur et son histoire. L'espace est donc social et, s'il peut finir sur une carte, il ne commence pas là.

\section{Les enjeux du développement social}

La lutte contre la pauvreté et les questions plus générales de la revitalisation de secteurs soulèvent donc à la fois des préoccupations économiques, mais en les 
incluant dans une trame où les activités de production de richesse se retrouvent liées à des activités qui les débordent. C'est dire que les activités de la Table de lutte à la pauvreté s'insèrent, de façon plus ou moins implicite, dans la perspective du développement économique communautaire. Trois types de difficultés ont été particulièrement notables : les relations entre les activités marchandes et les liens sociaux, la définition de l'espace public et la question de la polarisation sociale.

La question des rapports entre activités «marchandes » et les autres types d'activités est une question sensible pour les acteurs réunis dans la Table de lutte à la pauvreté. Cela tient en partie à leur inexpérience : peu d'entre eux ont réalisé ou réalisent des activités directement marchandes (vente de biens ou de services), et ceux qui le font les considèrent comme des aspects marginaux de leurs actions. Peu de ces groupes pratiquent la tarification de leurs services auprès de leurs usagers et encore moins visent à accumuler des bénéfices. Ils se conçoivent comme œuvrant selon une logique de solidarité, où les relations sont motivées par le besoin et la morale, non par le calcul. En fait, les activités à but lucratif ont bien mauvaise presse; elles sont vues comme plus ou moins immorales, car impliquant de l'égoïsme. Par extension, ceux qui les pratiquent sont perçus comme des exploiteurs peu enclins à des motivations altruistes et, s'ils en démontrent, ils sont soupçonnés de ne le faire qu'à des fins de relations publiques. Cela explique peut-être le peu d'empressement des participants à inclure des représentants des « milieux économiques » dans les activités de la Table. Selon les termes d'un intervenant, « on ne va pas demander aux riches de régler la pauvreté ».

Par contre, les organismes ont tous une expérience économique, car ils gèrent des salaires, des budgets, des subventions et des immobilisations. Le désintérêt envers l'économie lucrative ne doit donc pas être compris comme une désaveu des dimensions économiques de la vie sociale et organisationnelle. Il s'agit plutôt d'une volonté de se situer dans une économie publique ou solidaire faisant la place la plus large aux critères politiques et éthiques, où la notion de profit, avec ce qu'elle sous-entend d'appropriation inégale des surplus générés, s'insère assez mal. Il est possible que cela explique une partie des réticences des participantes (et sans doute du milieu communautaire en général) à l'égard de l'économie sociale.
L'espace social à l'intérieur duquel tentent de se situer les participantes à la Table de lutte à la pauvreté utilise des lignes de structuration qui se rattachent à la société civile. On y retrouve l'accent sur l'association et la concertation, modes de transaction qui diffèrent du marché et de la hiérarchie, ainsi qu'une recherche $\mathrm{du}$ consensus qui impose une approche dialogique plus qu'autoritaire ${ }^{19}$.

\section{Le désintérêt envers l'économie lucrative ne doit pas être compris comme une désaveu des dimensions économiques de la vie sociale et organisationnelle.}

Cet espace social (le terme signifie ici : ce qui se distingue de l'économique et du politique) est aussi un espace public, car il repose d'abord sur les organisations, qui sont vues comme ses acteurs premiers. On est donc au-delà de la stricte sphère privée qui met en lien des individus, mais sans être absorbé par l'étatique, ainsi que cela se fait souvent : la solidarité qui s'y manifeste est une solidarité de proximité, d'immédiateté et de situation partagée. Elle implique que la légitimité des acteurs en présence reposera non sur la délégation formelle, mais sur leur engagement, leur contact direct avec les enjeux de pauvreté et les personnes qui la vivent ${ }^{20}$.

\section{Conclusion : la démocratie, quotidienne et publique}

L'expérience de la Table de concert-action sur la lutte à la pauvreté de Chicoutimi donne quelques illustrations de ce que peut signifier la question du social à l'échelon local. Il en ressort que les responsabilités nouvelles des municipalités marquent une modification importante des relations entre l'État et la société civile, c'est-à-dire de l'intervention de la société sur elle-même. Beaucoup plus localisée que pendant les années de gloire de l'État-providence, celle-ci se fait parfois de façon parallèle aux institutions publiques. Elle montre que cette organisation (micro-)locale soulève la place des relations marchandes dans la définition des liens sociaux. Elle repose en bonne partie sur la façon de définir l'espace public et sur le mode de légitimation des acteurs en présence. De plus en plus, les institutions locales auront à tenir compte non seulement des paliers supérieurs de gouvernement, mais aussi des conditions de vie quotidiennes 
de leurs populations, une tâche rendue d'autant plus délicate par l'accentuation des processus de polarisation.

C'est là l'objet fondamental de la «question sociale » post-keynésienne : non seulement offrir des services à la population, mais offrir ce que la Marche des femmes de 1995 avait appelé des "infrastructures sociales ", c'est-à-dire des ressources permettant à la société civile, cette zone formée d'associations, de regroupements, de mouvements visant à générer du lien entre groupes et individus, de produire elle-même les conditions de son existence. On aura compris qu'elle n'est pas coupée de la vie marchande, qui est l'architecture essentielle de nos sociétés, ni de la vie politique, mais il est tout aussi clair qu'elle met en œuvre des principes différents. L'enjeu est de coordonner ces principes afin qu'ils s'épaulent plutôt que de s'annuler.

Cela signifie que le palier local sera un lieu essentiel des efforts de la société pour se produire et se contrôler elle-même, ce qui ne sera pas facile en des temps dominés par la mondialisation et les entreprises qui en sont les acteurs (et les bénéficiaires) premiers. Les approches formelles de la démocratie, qui délèguent à d'autres la légitimité de la parole publique, devront être « approfondies » ${ }^{21}$ et s'ouvrir à des principes de fonctionnement faisant une plus large place à l'autoorganisation. La démocratie quotidienne, c'est de faire avec les citoyens, plutôt que pour eux.

\section{Cela signifie que le palier local sera un lieu essentiel des efforts de la société pour se produire et se contrôler elle-même, ce qui ne sera pas facile en des temps dominés par la mondialisation et les entreprises qui en sont les acteurs (et les bénéficiaires) premiers.}

\section{Notes et références}

Le féminin est épicène, car les femmes sont en majorité.

2 Voir le compte-rendu dans : Tremblay, P.A., D. Martel, M. Tremblay et M. Alonso (1998). La pauvreté à Chicoutimi et à Jonquière : ce qu'on en dit, ce qu'on en fait, rapport de recherche remis à Développement économique Canada, Emploi-Québec, Société de développement de Jonquière et Société de développement économique de Chicoutimi, Chicoutimi, Collectif de recherche sur les ini- tiatives de développement économique et social (Collectif IDÉES-UQAC), 87 p. Et, pour un bilan partiel et daté : Tremblay, P.A., M. Alonso, M. Duplain et M. Pilote (2000). Bilan de la table de lutte à la pauvreté de Chicoutimi, rapport de recherche remis à Emploi-Québec, Chicoutimi, Collectif Idées-UQAC, 91 p.

4 B. Jessop (1994). " Post-fordism and the state », dans A. Amin (dir.), Post-fordism : A Reader, Oxford, Blackwell, p. 251-279. Jessop indique que le fordisme est centré sur le couple production de masse-consommation de masse. Le fordisme présente 5 dimensions : 1) procès de travail (ou de production), 2) régime d'accumulation, 3) mode social de régulation économique, 4) mode de sociétalisation; 5) formation sociale intégrant les 4 points précédents.

5 Castells, M. (1996). The rise of the network society, New York, Blackwell.

6 Urry, J. (2001). Sociology beyond societies, London, Routledge.

7 Beck, U. (1992). Risk society, Oxford, Polity press.

8 Ewald, F. (1996). Histoire de l'État-providence, Paris, Grasset, coll. Livre de poche.

9 Polanyi, K. (1944). The great transformation, Boston, Beacon press, 1957.

10 Néo-libéralisme: il s'agit de l'idéologie selon laquelle le retrait de l'État est la meilleure façon de régler les problèmes liés à l'épuisement du fordisme.

11 Décentralisation : « The transfer of responsibility for planning, management, and resource raising and allocation from the central government to : a) field units of central government ministries or agencies, b) subordinate units or levels of government, c) semi-autonomous public authorities or corporations, d) area-wide regional or functional authorities, or e) organizations of the private and voluntary sector ». Source : Rondinelli, D.A. (1981). Government «Decentralization in Comparative Perspective: Theory and Practice in Developing Countries ", International Review of Administrative Science, vol. 47.

12 Réingéniérie : le terme fait références aux volontés du gouvernement du Québec de réorganiser la production des services gouvernementaux en favorisant les partenariats public-privé.

13 Schetagne, Sylvain (1999) La pauvreté dans les régions métropolitaine du Québec. Rapport préliminaire de recherche, Ottawa, Conseil canadien de développement social.

${ }^{14}$ Torjman, S. et E. Leviten-Reid (2003). Le rôle social de l'administration locale, Ottawa, Caledon Institute of Social Policy.

${ }^{15}$ Gusfield, J. (1981). The culture of public problems, Chicago, Chicago Univ. Press.

16 Tremblay, P.A. et al. (1998), op.cit. 
17 Source : Tremblay, P.A., J. Rouffignat, M. Duplain et M.C. Clouston (2003). Atlas des conditions de vie et de la sécurité alimentaire au Saguenay-Lac-Saint-Jean. Analyse des interventions en sécurité alimentaire au Saguenay-LacSaint-Jean: les acteurs au cour du processus. Phase 1, 1 CD-Rom (96 cartes), rapport de recherche remis à la Direction de la santé publique, RRSSS-02, Chicoutimi, Collectif IDÉES-UQAC.

18 P.A. Tremblay (2003). «Qu'y a-t-il dans une communauté ? L'exemple des groupes communautaires québécois », Interventions économiques, vol. 30.

http:/www.teluq.uquebec.ca/ interventionseconomiques.
19 Ion, Jacques (1991). «Le politique et l'associatif», dans H. Drouard, M. Legros et H. Pascal (dir.), Sociologie et intervention sociale, Paris, Centurion, p. 232-248.

20 Cela peut expliquer pourquoi les représentants d'«organismes publics » (c'est-à-dire étatique) demeurés à la Table sont des intervenants de première ligne plutôt que des gestionnaires : leur proximité par rapport au «terrain » les rapproche des représentants des groupes communautaires.

21 Fung, A. et E.O. Wright (dir.) (2003). Deepening democracy. Institutional innovations in empowered participatory governance, London, Verso. 